\title{
The stability of dual-PSD based calibration system without its relative location to 3D forming of carbon fiber
}

\author{
Chengzhi Su ${ }^{1, a^{*}}$, Lei Zhong ${ }^{1, b}$, Chengyun Wang ${ }^{1, c}$, \\ Huzaifa Khalid $^{1, d}$, Fangchao Shao ${ }^{1}$ \\ ${ }^{1}$ College of Mechanical and Electric Engineering, Changchun University of Science and Technology, \\ Jilin Changchun 130022, China \\ achengzhi_su@126.com, b1532782478@qq.com, ’623935104@qq.com, \\ dhuzaifa.khalid@hotmail.com
}

Keywords: Carbon fiber, 3D forming robot, Calibration, Dual-PSD, Alignment, Lyapunov method

\begin{abstract}
The trajectory accuracy of the carbon fiber 3D forming robots is one of the key factors to ensure the quality of forming. It is well known that carbon fiber 3D forming robots have high repeatability but low accuracy. Compared to conventionally used calibration methods, dual-PSD based methods provide a more efficient way to accurately calibrate the joint offsets of industrial robots. But the stability of dual-PSD calibration system requires a strict proof in mathematics when the relationship between the robot base frame and the PSD frame is unknown. In this paper, by using an adaptive algorithm based on Lyapunov function, we can estimate the unknown parameters online without the parameters of the relationship. It is proved that the laser spots were stable to the desired positions on the dual-PSD plane. The performance is confirmed by the simulations at last. The carbon fiber 3D forming robots positioning accuracy can reach $0.0222 \mathrm{~mm}$ when the method proposed in this paper is used successfully. In this case, the quality of the forming can be assured.
\end{abstract}

\section{Introduction}

Carbon fiber is a new type of industrial material and has been applied in many fields. The carbon fiber 3D forming robots can print various precise parts, so its' accuracy is of critical importance. The high precision automatic calibration system is a hot research topic to improve the precision of 3D forming robot localization. Dual-PSD based robot calibration system has been proposed in reference [1]. Due to that the position of the dual-PSD in the robot's task space is unknown, the study on the stability of the unknown system is the primary problem of the calibration method. For the study on the stability of robot system, models and control approaches based on dual-PSD have been proposed by Yunyi Jia, Chengzhi Su, et al. [2,3] and the simulation experiments have been carried out. Zhihui Deng, Yunyi Jia, et al. [4] have designed the dual-PSD based adaptive alignment algorithm and have given a conclusion that the system is stable by simulation. Yantao Shen, Guoliang Xiang, et al. [5] have adopted visual servo control on a planar robot when the transformation matrix between the CCD camera frame and the robot base frame is unknown and have proved the stability of the system by Lyapunov method. In literature [6], Yantao Shen, Ning $\mathrm{Xi}$, et al. have proposed the adaptive algorithm when the position of the PSD array is unknown in the robot task space, eventually have reached the conclusion that the laser spots were stable to the desired positions. For the stability of the system, in the literature [2-4], the corresponding control algorithms have been proposed, and the simulation results have been demostrated, however, the theoretical proof has not been discussed. In literature $[5,6]$, the discussion of the system stability was only on planar robots. There is no theoretical proof about the stability of the space robot calibration system based on dual-PSD. The Lyapunov function based adaptive estimation law is designed and the large scale asymptotic stability of the calibration method have been proved in this paper, which lays a theoretical foundation for the study of the calibration system. 


\section{The Dual PSD Calibration System Overview}

The calibration configuration is shown in Fig. 1. The configuration includes a 6 degrees of freedom (DOF) manipulator to be calibrated, an industrial robot controller (IRC5 Compact), a laser pointer, a camera fixture and our position sensitive calibration fixture (PSCF). The semiconductor laser is mounted on its fixture and rigidly attached to the robot TCP. Two segmented PSDs are mounted on the two surface center of the PSCF as shown in Fig. 1. PSD is a high-precision photoelectric position sensor; it has a resolution of $0.1 \mu \mathrm{m}$.

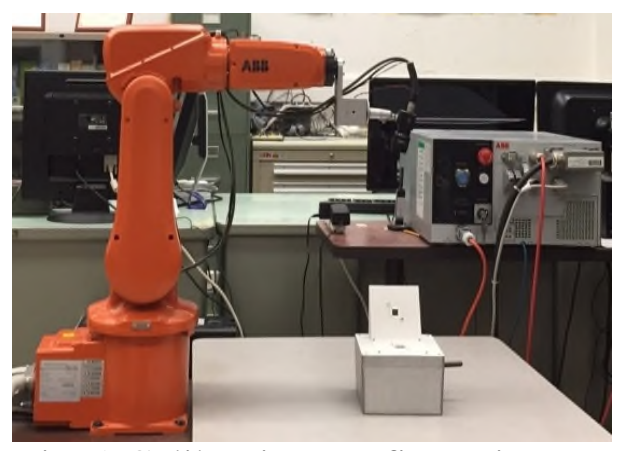

Fig. 1 Calibration configuration.

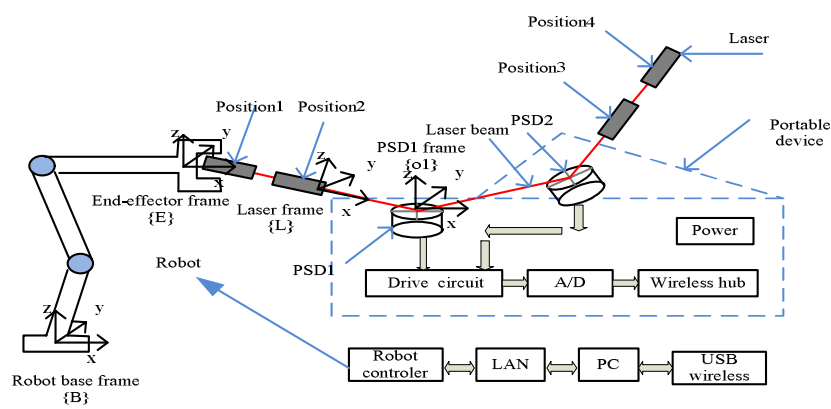

Fig. 2 Calibration scheme.

As shown in Fig. 2. The calibration method requires a laser beam to shoot at one of the PSDs at a certain angle and the reflected beam to shoot at the other PSD. Our servo control algorithm can make sure that the two laser beams tend to PSD center simultaneously. After the raw data of the spot position information is obtained by the PSD driver board, the signal from the data acquisition card is sent to the USB wireless hub. Once the data reaches the computer, the PC-based controller is used to make the two laser spots reach the desired position. Fig. 2 shows the definition of each Cartesian coordinate system. Frame $\sum o_{1}$ is built on the center of PSD1, frame $\sum B$ is the robot base frame. However, the relationship between $\sum o_{1}$ and $\sum B$ is unknown. The origin of $\sum L$ is defined in the center of the laser emission hole, the coordinate of the origin is $\left(p_{x}, p_{y}, p_{z}\right)$ with respect of frame $\sum o_{1}$, and laser line is defined as axis $\mathrm{x}$ in frame $\sum L \cdot \sum E$ is the robot end-effector frame.

\section{The Establishment of Mathematical Model for Robot Calibration}

Forward Kinematic Model from the Robot Joints to the Dual-PSD. As shown in Fig. 3, $o_{1}, o_{2}$ are the center points of the 2 PSD planes and are the coordinate origins of frame $\sum o_{1}$ and $\sum o_{2} \cdot \gamma$ is the angle between the PSD1 plane and the PSD2 plane. $d / 2$ is the distance from $o_{1}$ to the insection of PSD1 plane and PSD2 plane. $l_{1}$ is the incident line of the PSD1 plane and the intersection of $l_{1}$ and PSD1 plane is defined as $p_{1}, p_{1} p_{2}$ and $l_{2}$ represent the reflected line of the PSD1 plane and the PSD2 plane respectively.

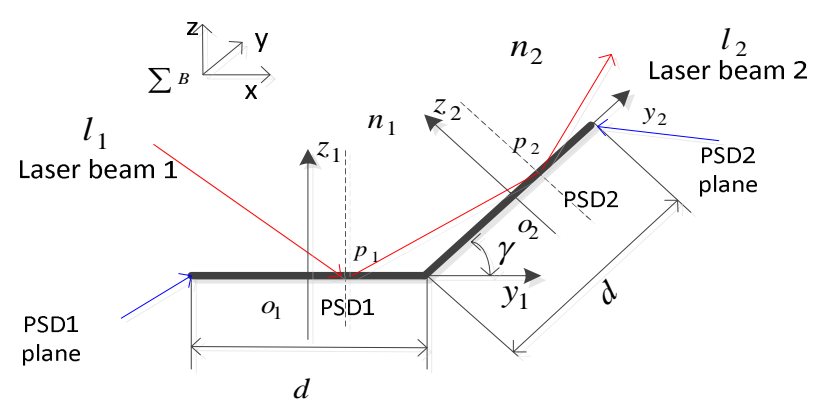

Fig. 3 The dual PSD calibration device. 
${ }_{B}^{o_{1}} T$ is the pose matrix of frame $\sum B$ under the frame $\sum o_{1}$ and ${ }_{L}^{E} T$ is the pose matrix of frame $\sum L$ under the frame $\sum E$, they are shown as follows:

$$
{ }_{B}^{a_{1}} T=\left[\begin{array}{cccc}
k_{1} & k_{2} & k_{3} & k_{4} \\
k_{5} & k_{6} & k_{7} & k_{8} \\
k_{9} & k_{10} & k_{11} & k_{12} \\
0 & 0 & 0 & 1
\end{array}\right] \text { and }{ }_{L}^{E} T=\left[\begin{array}{cccc}
u_{11} & u_{12} & u_{13} & u_{14} \\
u_{21} & u_{22} & u_{23} & u_{24} \\
u_{31} & u_{32} & u_{33} & u_{34} \\
0 & 0 & 0 & 1
\end{array}\right]
$$

Because the space relationship between frame $\sum o_{1}$ and frame $\sum B$ is unknown, $k_{1} \sim k_{12}$ is the unknown parameters, while ${ }_{L}^{E} T$ is already known. $\vec{p}_{1}=\left[{ }^{o_{1}} x_{1},{ }^{o_{1}} y_{1}{ }^{o_{1}} z_{1}\right]^{T}$ and $\vec{p}_{2}=\left[{ }^{o_{1}} x_{2},{ }^{o_{1}} y_{2}{ }^{o_{1}} z_{2}\right]^{T}$ denote the coordinate vectors of $p_{1}$ and $p_{2}$ respectively under the frame $\sum o_{1} . Y=\left[{ }^{o_{1}} x_{1},{ }^{o_{1}} y_{1},{ }^{o_{1}} x_{2},{ }^{o_{1}} y_{2}\right]^{T}$ is the output vector of PSCF. $q=\left[q_{1}, q_{2}, q_{3}, q_{4}, q_{5}, q_{6}\right]^{T}$ is the robot joint vector.

Assuming that the unit directional vector of the Laser line $l_{1}$ is $\vec{l}_{1}=\left[n_{x}, n_{y}, n_{z}\right]^{T}$, and the position and orientation vector of the $\mathrm{x}$-axis of frame $\sum L$ is ${ }_{l_{1}}^{o_{1}} U=\left[n_{x}, n_{y}, n_{z}, p_{x}, p_{y}, p_{z}\right]^{T}$. The coordinate of $p_{1}$ can be expressed as:

$$
\left\{\begin{array}{l}
{ }^{o_{1}} x_{1}=n_{x} t_{1}+p_{x} \\
{ }^{o_{1}} y_{1}=n_{y} t_{1}+p_{y} \\
{ }^{{ }^{o_{1}} z_{1}}=0
\end{array}\right.
$$

$t_{1}$ denotes the distance between the origin of frame $\sum L$ and $p_{1}$, and can be measured by the Laser triangulation. The coordinate of $p_{2}$ can be expressed as:

$$
\left\{\begin{array}{l}
{ }^{o_{1}} x_{2}=n_{x}\left(t_{1}-t_{2}\right)+p_{x} \\
{ }^{o_{1}} y_{2}=n_{y}\left(t_{1}-t_{2}\right)+p_{y} \\
{ }^{o_{1}} z_{2}=n_{z} t_{2}
\end{array}\right.
$$

$t_{2}$ denotes the distance between $p_{1}$ and $p_{2}$, and can be obtained by the distance formula between two points. The expression of $Y$ can be obtained by equation group(2)and(3). The derivative of $Y$ is

$$
\dot{Y}=J^{\prime o_{1}} \dot{U}
$$

The kinematics model was established by using the method of DH[10,11], and the pose matrix of frame $\sum E$ under the frame $\sum B$ which can be written ${ }^{B}{ }_{E}^{B} T$ is derived. The pose matrix of frame $\sum L$ under the frame $\sum o_{1}$ is

$$
{ }_{L}^{o_{1}} T={ }_{B}^{o_{1}} T_{E}^{B} T_{L}^{E} T=\left[\begin{array}{cccc}
n_{x} & o_{x} & a_{x} & p_{x} \\
n_{y} & o_{y} & a_{y} & p_{y} \\
n_{z} & o_{z} & a_{z} & p_{z} \\
0 & 0 & 0 & 0
\end{array}\right]
$$

Due to space limitations, only to show $n_{x}\left(s i, c i\right.$ denotes $\sin \left(q_{i}\right), \cos \left(q_{i}\right)$ respectly, $s 23, c 23$ denotes $\sin \left(q_{2}+q_{3}\right), \cos \left(q_{2}+q_{3}\right)$ respectly):

$$
\begin{aligned}
& n_{x}=u_{21}\left(k_{3}\left(s_{6}\left(c_{23} s_{5}+c_{4} c_{5} s_{23}\right)+c_{6} s_{4} s_{23}\right)-k_{1}\left(s_{6}\left(c_{5}\left(s_{1} s_{4}+c_{1} c_{4} c_{23}\right)-c_{1} s_{5} s_{23}\right)-c_{6}\left(c_{4} s_{1}-c_{1} c_{23} s_{4}\right)\right)\right. \\
& \left.+k_{2}\left(s_{6}\left(c_{5}\left(c_{1} s_{4}-c_{4} c_{23} s_{1}\right)+s_{1} s_{5} s_{23}\right)-c_{6}\left(c_{1} c_{4}+c_{23} s_{1} s_{4}\right)\right)\right)-u_{11}\left(k_{3}\left(c_{6}\left(c_{23} s_{5}+c_{4} c_{5} s_{23}\right)-s_{4} s_{6} s_{23}\right)\right. \\
& -k_{1}\left(c_{6}\left(c_{5}\left(s_{1} s_{4}+c_{1} c_{4} c_{23}\right)-c_{1} s_{5} s_{23}\right)+s_{6}\left(c_{4} s_{1}-c_{1} c_{23} s_{4}\right)\right)+k_{2}\left(c_{6}\left(c_{5}\left(c_{1} s_{4}-c_{4} c_{23} s_{1}\right)+s_{1} s_{5} s_{23}\right)+s_{6}\left(c_{1} c_{4}\right.\right. \\
& \left.\left.\left.+c_{23} s_{1} s_{4}\right)\right)\right)-u_{31}\left(k_{1}\left(s_{5}\left(s_{1} s_{4}+c_{1} c_{4} c_{23}\right)+c_{1} c_{5} s_{23}\right)-k_{2}\left(s_{5}\left(c_{1} s_{4}-c_{4} c_{23} s_{1}\right)-c_{5} s_{1} s_{23}\right)+k_{3}\left(c_{5} c_{23}-c_{4} s_{5} s_{23}\right)\right)
\end{aligned}
$$

The derivative of ${ }_{l_{1}}^{o_{1}} U$ is

$$
{ }_{l_{1}}^{o_{1}} \dot{U}=J^{\prime \prime} \dot{q}
$$

and $J^{\prime \prime}=\left[\frac{\partial n_{x}}{\partial q}, \frac{\partial n_{y}}{\partial q}, \frac{\partial n_{z}}{\partial q}, \frac{\partial p_{x}}{\partial q}, \frac{\partial p_{y}}{\partial q}, \frac{\partial p_{z}}{\partial q}\right]^{T}$ 
Combining Eq. (4) and (7), the forward kinematics model from the robot joints to the dual-PSD is derived:

$$
\dot{Y}=J \dot{q}=J^{\prime} \cdot J^{\prime \prime} \dot{q}
$$

The unknown parameters of the formula (9) are placed in the $12 \times 1$ column vector $\theta$, so that the known partial and unknown parameters are completely separated. That is

$$
\dot{Y}=J \dot{q}=D_{1} \theta
$$

Robotic Dynamic Model. The dynamic model of the robot with 6 degrees of freedom is estimated using Newton-Euler method; it follows the robot dynamics equation:

$$
H(q) \ddot{q}+C(q, \dot{q}) \dot{q}+G(q)=\tau
$$

where, $H(q)$ is the symmetric and positive definite inertia matrix. $C(q, \dot{q})$ denotes a skew-symmetric matrix. $G(q)$ is the gravity force. The $\tau$ represents the joint input of the manipulator.

\section{The Proof of Asymptotic Stability based on the Lyapunov Equation}

The Establishment of the Lyapunov Equation. In order to prove that the laser spots tend to the desired positions on the double PSD plane, the Lyapunov function is defined based on energy method:

$$
V=\frac{1}{2}\left\{\Delta \dot{q}^{T} H(q) \Delta \dot{q}+\Delta \theta^{T} B_{1} \Delta \theta+\Delta Y^{T} K_{p} \Delta Y\right\}
$$

$B_{1}, K_{p}$ is positive constant, $\hat{\theta}$ is an estimated value of $\theta$ and $\Delta \theta=\theta-\hat{\theta} ; \Delta q=q-q_{d}$ represents the joint vector errors; $Y_{d}$ represents the desired positions, $\Delta Y=Y-Y_{d}$ represents the position errors of laser spots, at this point $\Delta \dot{Y}=\dot{Y} ;$ also, we have $\Delta \dot{q}=\dot{q}$.

The derivative of Eq. (12) is:

$$
\dot{V}=\dot{q}^{T} H \ddot{q}+\frac{1}{2} \dot{q}^{T} \dot{H} \dot{q}+\Delta \theta^{T} B_{1} \Delta \dot{\theta}+\Delta \dot{Y}^{T} K_{p} \Delta Y
$$

According to the characteristics of robot dynamics, $\dot{H}-2 C$ is skew-symmetric matrix, namely $\dot{H}=2 C$, so we have:

$$
\begin{aligned}
\dot{V} & =\dot{q}^{T} H \ddot{q}+\dot{q}^{T} C(q, \dot{q}) \dot{q}+\Delta \theta^{T} B_{1} \Delta \dot{\theta}+(J \dot{q})^{T} K_{p} \Delta Y \\
& =\dot{q}^{T}\left[H \ddot{q}+C(q, \dot{q}) \dot{q}+K_{p} J^{T} \Delta Y\right]+\Delta \theta^{T} B_{1} \Delta \dot{\theta}
\end{aligned}
$$

Combining (11) we have: $\dot{V}=\dot{q}^{T}\left[\tau-G(q)+K_{p} J^{T} \Delta Y\right]+\Delta \theta^{T} B_{1} \Delta \dot{\theta}$

The Design of Adaptive Control Law. The control problem belongs to the fixed point control, we can adopt PD plus gravity compensation algorithm to design the adaptive control law:

$$
\tau=G(q)-K_{v} \dot{q}-K_{p} \hat{J}^{T} \Delta Y
$$

$K_{v}$ is the positive constant, $\hat{J}^{T}$ represents the estimated matrix of $J^{T}$.Substituting the adaptive control law (16) into the robot dynamics Eq. (11), closed-loop dynamics equation is derived:

$$
H(q) \ddot{q}+C(q, \dot{q}) \dot{q}=-K_{v} \dot{q}-K_{p} \hat{J}^{T} \Delta Y
$$

The error between the real and the estimated value of the spot speeds:

$$
\dot{Y}-\dot{\hat{Y}}=(J-\hat{J}) \dot{q}=D_{1} \Delta \theta
$$

The Design of Adaptive Parameter Estimation Law. Substituting the designed control law (16) into Eq. (15), and combining Eq. (10), we can derive that 


$$
\dot{V}=-\dot{q}^{T} K_{v} \dot{q}+K_{p} \Delta \theta^{T} D_{1}^{T} \Delta Y+\Delta \theta^{T} B_{1} \Delta \dot{\theta}
$$

To get a stable system and make $\dot{q}$ and $\Delta \theta$ asymptotically stable to the desired value, an adaptively estimated law is designed as

$K_{p} \Delta \theta^{T} D_{1}^{T} \Delta Y+\Delta \theta^{T} B_{1} \Delta \dot{\theta}=-\Delta \theta^{T} B_{1} B_{2} \Delta \theta$

$B_{2}$ is a positive constant. Simplifying Eq. (20), the adaptively estimated law can be written as:

$$
\frac{d \Delta \theta}{d t}=-B_{2} \Delta \theta-\frac{K_{p}}{B_{1}} D_{1}^{T} \Delta Y
$$

The Proof of Asymptotic Convergence. The expression (21) is first order differential equation group with constant coefficients, set $a(t)=-\frac{K_{p}}{B_{1}} D_{1}^{T} \Delta Y$, then the solution of the equation group is:

$$
\Delta \theta=e^{A t} \Delta \theta_{0}+\int_{0}^{t} e^{A(t-\tau)} a(\tau) d \tau
$$

$\Delta \theta_{0}$ is the initial value of $\Delta \theta, A=-B_{2} E_{12 \times 12}, E_{12 \times 12}$ is 12 order unit matrix. When $t \rightarrow \infty$, in expression (22), $e^{A t} \Delta \theta_{0} \rightarrow 0$; so

$$
\lim _{t \rightarrow \infty} \Delta \theta=\lim _{t \rightarrow \infty} \int_{0}^{t} e^{A(t-\tau)} a(\tau) d \tau=\lim _{t \rightarrow \infty} e^{A t} \int_{0}^{t} e^{-A \tau} a(\tau) d \tau=\lim _{t \rightarrow \infty} \frac{a(t)}{B_{2}}
$$

The stability condition of estimation law: we can see from formula (10) that when $\dot{q}=0$, each element of matrix $D_{1}$ equals0, namely $a(t)=0$, at this point $\Delta \theta$ asymptotically stable to 0 .

Substituting Eq. (20) into Eq. (19),we can get:

$$
\dot{V}=-\dot{q}^{T} K_{v} \dot{q}-\Delta \theta^{T} B_{1} B_{2} \Delta \theta
$$

It is proved that there are global positive-definite Lyapunov function $V$, the differential of which is nonpositive. Set $\dot{V} \equiv 0$, at this point, $\dot{q} \equiv 0, \Delta \theta \equiv 0$ and only the zero solution satisfies the equation. According to LaSalle theorem, $\dot{q}=0$ is asymptotically stable, namely each element of matrix $D_{1}$ asymptotically stable to 0 , so $\hat{\theta}$ is bounded; so the derived stable value of $\dot{q}$ and $\Delta \theta$ is consistent with the stability condition of estimation law we have obtained. Combining Eq. (16), the conclusion is reached that the laser spot position error $\Delta Y$ on dual-PSD plane asymptotically stable to 0 when time tends to infinity.

\section{Simulation Experiments}

Materials. The density of the carbon fiber is $1.6-2.5 \mathrm{~g} / \mathrm{cm}^{3}$. It has small thermal expansion coefficient, good thermal conductivity, good wear resistance and resistance to chemical corrosion. The performance of carbon fiber is very stable at 400 degrees, so it has a good performance of high-temperature resistance.

Methods. To verify the validity of our calibration method, we show the stability of our calibrated system based on dual-PSD by simulations. The block diagram is shown in Fig. 4. For the simulation, the following data is used: positive constant $K_{p}=0.0005, K_{v}=0.8, B_{1}=100, B_{2}=0.000001$; Set initial value $\Delta Y_{0}=[4,-4,4,-4]^{T}$; the rotation matrix of ${ }_{L}^{E} T$ is unit matrix, and the position vector is ${ }_{L}^{E} p=[10,10,10]^{T}$; the real rotation matrix is derived by rotating $\frac{\pi}{6}, \frac{\pi}{6}, \frac{\pi}{6}$ around axis $x, y, z$ of frame $o_{1}$ respectively and the position vector is given ${ }_{B}^{o_{1}} p_{\text {real }}=[100,100,60]^{T}$. The initial matrix of ${ }_{B}^{o_{1}} T$ is equal to the real one and the initial vector $\Delta \theta_{0}$ is set to 0 in this simulation. Fig. 5 shows the simulation results, we can see that the laser position errors tend to zero quickly and the estimated values $\hat{\theta}$ tend to the real ones with the time increases. According to the simulation experiments, the system is large scale asymptotic stable. The part data of simulation is shown in Table 1 . The 
robots positioning accuracy can reach $0.0222 \mathrm{~mm}$.

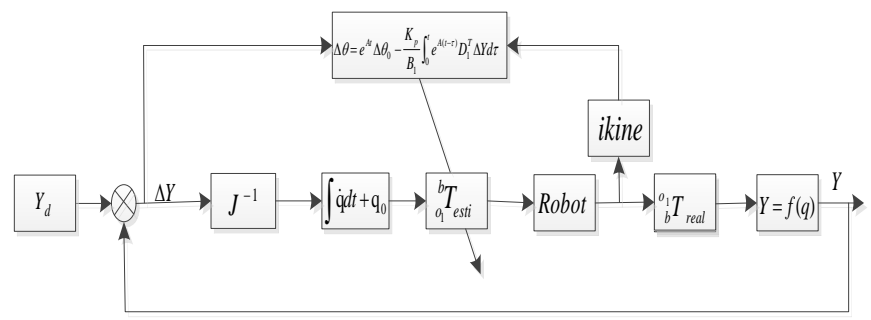

Fig. 4 The closed-loop servo control model.

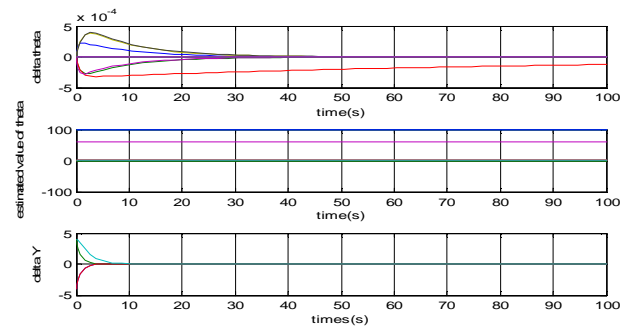

Fig. 5 Position errors of laser points.

Table 1. Various initial values and its' steady-state errors.

\begin{tabular}{c|c|c}
\hline & Initial value $[\mathrm{mm}]$ & Steady-state error $[\mathrm{mm}]$ \\
\hline 1 & $Y_{0}=[4,-4,4,-4]^{T}$ & $Y_{\text {error }}=[0.0104,0,0.0164,0]^{T}$ \\
\hline 2 & $Y_{0}=[-4.1,4,-5,4]^{T}$ & $Y_{\text {error }}=[0.0141,0,0.0222,0]^{T}$ \\
\hline 3 & $Y_{0}=[-4,4.7,-4,3.7]^{T}$ & $Y_{\text {error }}=[0.0095,0,0.015,0]^{T}$ \\
\hline 4 & $Y_{0}=[-2.1,4.75,15.5,3.7]^{T}$ & $Y_{\text {error }}=[0.0048,0,0.007,0]^{T}$ \\
\hline 5 & $Y_{0}=[-1,9,74,-66]^{T}$ & $Y_{\text {error }}=[0.0032,0,0.0057,0]^{T}$ \\
\hline
\end{tabular}

\section{Summary}

When the relationship between the carbon fiber 3D forming robot base frame and PSD frame is unknown, the stability to make the laser beam align to the dual-PSD centers simultaneously is an important problem in improving the robot accuracy area. In this paper, the kinematics and dynamics models of the robot are established, and the system stability is proved based on Lyapunov equation. The simulation experiments have proved that the laser spots stable to the desired positions .The carbon fiber 3D forming robots positioning accuracy can reach $0.0222 \mathrm{~mm}$ using our method, and the quality of the forming is improved comparing to the accuracy of $0.08 \mathrm{~mm}$ before the calibration.

\section{Acknowledgements}

Supported by International Science and Technology Cooperation Fund Project of Jilin Province (20160414030GH)

\section{Reference}

[1] E. Nieves, N. Xi, B. Du, Y. Jia, A Reflected Laser Line Approach for Industrial Robot Calibration, IEEE/ASME Int. Conf. Adv. Intell. Mech. (2012) 610-615.

[2] Y. Jia, C. Su, et al. Multi-objective Position Control for an Industrial Robot Calibration system, IEEE Int. Conf. Autom. Sci. Eng. (2015) 1236-1241.

[3] C. Z. Su, L. Zhong, et al, Modelling and Control for Simultaneous Laser Beam Alignment of a Dual-PSD Industrial Robot Calibration System. The 5th Annual IEEE International Conference on Cyber Technology in Automation, Control and Intelligent Systems. (2015) 902-907.

[4] Z. H. Deng, Y. Jia.et al, Adaptive Alignment Control for a Dual-PSD based Industrial Robot Calibration System, IEEE Conf. Robot. Biom. (2015) 1247-1252.

[5] Y. Shen, G. Liang, Y. H. Liu, Uncalibrated Visual Servoing of Planar Robots, IEEE Robot. Autom. (2002) 580-585.

[6] Y. Shen, N. Xi, X. Z. Li, et al, Robot Localization Using an Uncalibrated PSD Servoing Approach, the 7th World Congress on Intelligent Control and Automation. (2008) 535-540. 